\title{
Contingent learning
}

\author{
HERMAN BUSCHKE \\ Saul R. Korey Department of Neurology, Rose F. Kennedy Center for Research in Mental Retardation \\ and Human Development, and Department of Neuroscience, Albert Einstein College of Medicine, \\ Bronx, New York 10461
}

Individual subjects were allowed to learn at their own rates by adding more items on each trial, contingent on current recall, while reminding them of any items not recalled to assure learning of all items presented. The constantly increasing recall in such ongoing learning was sustained by remembering more items as part of larger recall units.

In most studies of human verbal learning, it has been customary to present a fixed number of items on every trial, which may not be necessary (Buschke \& Hinrichs, 1968; Jung, 1964; Mandler \& Dean, 1969) or desirable, since it limits learning and makes it difficult to determine how rapidly an individual can learn. This report describes the ongoing learning obtained when individuals are allowed to learn at their own rates, by adding more items on each trial contingent on current recall, and shows that the constant increase of recall in such continuous learning is due to remembering more items as part of larger units (Bower, 1970; Miller, 1956) that were identified by two-dimensional recall (Buschke, 1977). This procedure accounts for the rate of learning in terms of the increasing number of items remembered at the same time as part of a cluster, as well as the number of items remembered as part of a sequence, indicating the value of identifying the units of recall involved in the process of remembering.

\section{METHOD}

Each of 10 young adult subjects learned up to 60 items from two sets of common unrelated four- or five-letter words in a counterbalanced design with an interval of 2 months between first and second testing. Each subject was tested individually. The items were presented by reading them aloud to the subject at a 2-sec rate. Immediately after presentation, the subject recalled all current and previously presented items aloud in any order, concurrent with the written two-dimensional recall to be described. Enough time and encouragement were provided to maximize recall on each trial (Buschke, 1973, 1974). On the first trial, 20 items were presented. On subsequent trials, the subject was reminded of all items not recalled on the immediately preceding trial and, if less than 10 reminders were needed, a number of new items equal to 10 minus the number of reminders was presented. (For example, if eight were needed only two new items were added, while if only four reminders were needed six new items were added.) Except for the first trial (and any rare trials on which more than 10 reminders were

This work was supported in part by PHS Grants MH-17733 from NIMH, NS-03356 from NIMS, and HT-01799 from NICHD. I thank Christine Sinclair-Prince for experimental assistance. Requests for reprints should be sent to Herman Buschke, Department of Neurology, Albert Einstein College of Medicine, 1300 Morris Park Avenue, Bronx, New York 10461. needed), 10 items were presented on each trial, varying from 10 reminders and no new items to no reminders and 10 new items. Unlike the noncontingent presentation of just one new item on every trial (Mandler \& Dean, 1969), the present procedure is intended to obtain an estimate of the rate or efficiency of ongoing individual learning by providing enough additional contingent presentation to allow each individual to learn at his own rate, while selectively reminding him of any items not recalled (Buschke, 1973) to assure learning of all previously presented items and to confirm learning by recall without continuing presentation. Free recall of unrelated words was used because it allows spontaneous organization of items that are not already organized.

\section{RESULTS AND DISCUSSION}

\section{Rate of Learning}

Figure 1 (left) shows the mean number of items recalled on each trial of first and second testing, the number recalled without presentation on that trial, the

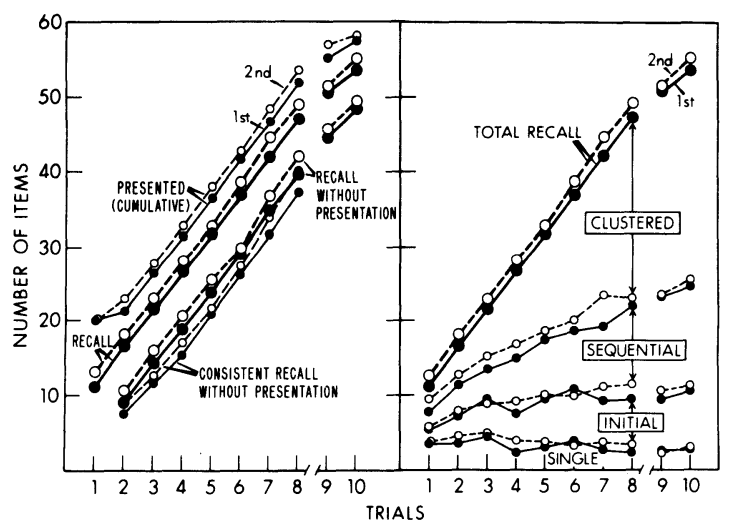

Figure 1. Left: Linear recall functions in ongoing learning on first and second testing of 10 young adults. The curves are broken after Trial 8 because not enough new items were left for complete additional presentation after that trial. Right: Changing composition of constantly increasing recall during ongoing learning shown by complete two-dimensional recall. The arrows show how the components of recall were added up to account for the total number of items recalled on each trial: Items recalled by themselves (single), initial items of sequences or clusters (initial), additional items recalled as part of a sequence (sequential), and additional items recalled as part of a cluster of simultaneously remembered items (clustered). 
number recalled consistently on all subsequent trials without any further presentation, and the cumulative number of different items presented for learning. The curves are broken after Trial 8 because there were not enough new items for complete additional presentation after that trial. The cumulative number of presented items, which shows the maximum recall possible on each trial, is correlated with recall because the number of additional items presented on each trial is contingent on the immediately preceding recall. Although presentation of more than 10 additional items on each trial should show a faster rate of learning, since it is well known that more may be recalled from longer lists (Shiffrin, 1970), it appears that presentation of up to 10 additional items on each trial was sufficient to allow maximum individual recall under the present experimental conditions, since recall was less than perfect.

The rate of learning on first and second testing was very similar. The recall functions in Figure 1 (left) appear to be linear, increasing at an approximately constant rate as long as there were enough items for compete additional presentation. The mean rate of learning, calculated by averaging the slopes of the bestfitting linear function for recall by each individual over Trials $1-8$, was $5.13(\mathrm{SD}=1.02)$ items per trial on first testing and $5.21(\mathrm{SD}=1.02)$ on second testing; these rates were not significantly different $(t=.38)$. Most of the items were recalled without presentation from Trial 2 on. Recall without presentation also appears linear. The rate of learning shown by recall without presentation over Trials 1.8 was $5.14(\mathrm{SD}=1.03)$ items per trial on first testing and $5.22(\mathrm{SD}=1.10)$ on second testing, which were not significantly different $(t=.25)$ and were close to the rates of learning obtained from total recall. The number of items recalled with presentation, including both reminders and new presentations, remained fairly constant, as shown by the difference between total recall and recall without presentation. Most of the items recalled without presentation were recalled consistently on all subsequent trials without any further presentation, which is the strictest criterion of learning and may provide the best indication of how much has been learned (Buschke, 1973; Fuld \& Buschke, 1976). The rate of learning shown by consistent recall without presentation was $5.01(\mathrm{SD}=1.22)$ on first testing and $5.25(\mathrm{SD}=1.16)$ on second testing, which also were not significantly different $(t=.58)$. Since the learning rates on first and second testing did not differ significantly, it appears that this paradigm can provide a reliable estimate of individual learning rate under conditions that permit each individual to learn at his own rate. This procedure yields a reasonable measure of ongoing learning without either overwhelming slower learners or underestimating the capacity of faster learners, which should be useful for studies of learning in development, aging, dementia, learning disabilities, and individual differences.
Although the linear recall functions shown in Figure 1 (left) appear to be very different from the typical negatively accelerated or exponential learning curves found in standard multitrial free recall (Murdock, $1960,1974)$ when the same set of items is presented on every trial, the constant increase of recall in ongoing learning can be explained on the same basis as the negatively accelerated recall functions. Negatively accelerated recall is usually attributed to a constant probability of recalling items not already recalled, together with continuing recall of items already recalled (Murdock, 1974, pp. 199-200). Since the number of items not already recalled decreases during multitrial free recall of a fixed number of items, the recall function will increase more slowly as learning proceeds. In the ongoing contingent learning described here, the number of items presented on each trial remained constant on Trials 2-8 and most of the items recalled without presentation were recalled consistently (Figure 1, left), resulting in a constant increase of recall. These subjects recalled about $50 \%$ of the presented items, including the 20 presented on Trial 1 as well as the 10 presented on Trials 2-8.

\section{Sequential and Simultaneous Remembering}

To analyze the changing composition of recall during ongoing learning and determine whether the continuing increase of recall is due to remembering more items as part of larger units, two-dimensional recall was used to show the components and organization of remembering on each trial (Buschke, 1977). A more complete form of two-dimensional recall was used to discriminate between sequential recall of items remembered as part of a sequence and clustered recall of several items remembered at the same time. To determine whether subjects might remember more than one item at a time, they were asked to write simultaneously remembered items across a single line of a two-dimensional response grid to show clustered recall, as illustrated in Figure 2. To show sequential recall, the subjects were asked to write items (or clusters) on successive lines when they were remembered as part of a sequence, skipping a line between items (or clusters) that were not connected in the process of remembering. ${ }^{1}$ The distinction between clustered and sequential recall may be important for analysis of remembering, since the recall of items as part of a cluster may involve retrieval of just one (larger) unit, while recall of items as part of a sequence may involve directed searching (Shiffrin \& Atkinson, 1969') for each item in terms of the preceding items.

Figure 1 (right) shows how the composition of recal changed during ongoing learning, sustaining the constan 1 increase of recall on both first and second testing by recall of more and more items as part of sequences anc clusters. Very few items were recalled by themselves and the small number of single items did not increase Recall of the initial item of sequence or clusters (set 


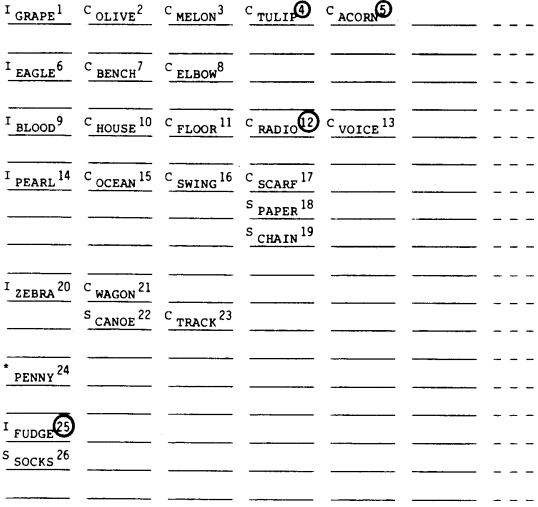

Figure 2. Typical protocol of complete two-dimensional recall, showing the composition and organization of recall by a typical subject on Trial 4 of ongoing learning. Numbers show the order of recall; circled numbers show items recalled for the first time on this trial. Letters identify the components of remembering shown by complete two-dimensional recall: single item $\left({ }^{*}\right)$, initial items of clusters or sequences (I), additional items recalled as part of a sequence (S), and additional items recalled as part of a cluster (C) of simultaneously remembered items.

Figure 2), shown added to single item recall in Figure 1 (right), increased slightly. Initial and single items together show the total number of "pieces" in recall. Most of the continuing increase of recall was due to recall of an increasing number of additional items remembered as part of a sequence or cluster; this analysis includes all items recalled as part of sequences or clusters on each trial, regardless of whether the same sequences or clusters occurred repeatedly or not. Recall of items as part of clusters of simultaneously remembered items accounted for an increasingly greater part of recall as learning proceeds. Most subjects showed predominantly clustered recall, but several subjects showed predominantly sequential recall, which may reflect differences in strategy. The number of additional sequential and clustered items also provides a measure of the organization due to sequencing and clustering, since the number of extra items recalled as part of a sequence or cluster is equal to the minimum number of $\mathrm{n}-1$ links needed to connect all of the $\mathrm{n}$ items in any sequence or cluster, the sum of which provides the simplest measure of organization based on the actual grouping (Buschke, 1977). Figure 1 (right) shows that the increasing amount of such organization is closely related to the increasing amount of recall.

Two-dimensional recall, which has been shown not to induce the units and organization it reveals (Buschke, 1977), shows that all but a few items are recalled as part of sequences or clusters, and that the increasing recall in ongoing learning is accounted for by the increasing recall of more items as part of sequences and clusters, demonstrating the role of organization in learning. Complete two-dimensional recall shows that much of recall apparently involves the recall of several items simultaneously, which could be due to recoding several items into a single cluster, or to multiple parallel retrieval searches, or both. Repeated recall of the same items simultaneously would seem to indicate recoding, while simultaneous recall of different items on each trial would seem to indicate parallel searching. In view of the general assumption that recall involves the retrieval of one thing at a time, recoding may seem more likely. Since this would mean that much of remembering involves recall of clusters rather than single items, it would seem appropriate to determine what the units of remembering are on each trial, so that the process as well as the results of remembering can be analyzed, in terms of the units actually involved in remembering (Tulving, 1968 , p. 29). Perhaps the most significant issue raised by the use of two-dimensional recall concerns the identification of such cognitive units, which have also been found in semantic remembering of text (Buschke $\&$ Schaier, in press) and recall of the items in a category from semantic memory (Buschke, 1977). Twodimensional recall can be used to identify the units of recall and delineate their organization on each trial, permitting analysis of learning and memory in terms of the units involved in remembering.

\section{REFERENCES}

Bower, G. H. Organizational factors in memory. Cognitive Psychology, 1970, 1, 18-46.

BUSCHKE, H. Selective reminding for analysis of memory and learning. Journal of Verbal Learning and Verbal Behavior, 1973, 12, 543-550.

BuschKe, H. Spontaneous remembering after recall failure. Science, 1974, 184, 579-581.

BusCHKE, H. Two-dimensional recall: Immediate identification of clusters in episodic and semantic memory. Journal of Verbal Learning and Verbal Behavior, 1977, 16, 201-215.

BuschKe, H., \& HinRichs, J. V. Controlled rehearsal and recall order in serial list retention. Journal of Experimental Psychology, 1968, 78, 502-509.

Buschke, H., \& Schaier, A. Memory units, ideas, and propositions in semantic remembering. Journal of Verbal Learning and Verbal Behavior, in press.

Fuld, P. A., \& BuschKe, H. Stages of retrieval in verbal learning. Journal of Verbal Learning and Verbal Behavior, 1976, 15, 401-410.

Jung, J. A cumulative method of paired-associate and serial learning. Journal of Verbal Learning and Verbal Behavior, 1964, 3, 290-299.

Mandler, G., \& Dean, P. J. Seriation: Development of serial order in free recall. Journal of Experimental Psychology, 1969, 81, 207-215.

Miller, G. A. The magical number seven, plus or minus two: Some limits in our capacity for processing information. Psychological Review, 1956, 63, 81-97.

MuRDock, B. B. The immediate retention of unrelated words. Journal of Experimental Psychology, 1960, 60, 222-234.

Murdock, B. B. Human memory. Potomac, Md: Erlbaum, 1974. Shiffrin, R. M. Forgetting: Trace erosion or retrieval failure? Science, 1970, 168, 1601-1603.

Shiffrin, R. M., \& Atkinson, R. C. Storage retrieval processes in long-term memory. Psychological Review, 1969, 76, 179-193.

Tulving, E. Theoretical issues in free recall. In T. R. Dixon \& D. L. Horton (Eds.), Verbal behavior and general behavior theory. Englewood Cliffs, N.J: Prentice-Hall, 1968. 


\section{NOTE}

1. The original version of two-dimensional recall (Buschke, 1977) only asked subjects to write items that were remembered "together" across a single line, which did not discriminate between sequential and simultaneous recall and did not delineate either the relations of successive items and groups or the internal structure of the groups. The present instructions for twodimensional recall allow the subject to show all possible relations among items and larger units in the process of remembering. To show the internal structure of clusters of simultaneously recalled items, these subjects also were asked to skip a blank between simultaneously recalled items that were not connected; the aim of this instruction was to discriminate between concurrent recall of items as part of an "associative" cluster containing related but distinct parts and recall of items incorporated as part of a "unitary" cluster without distinct parts. Almost all of the simultaneously remembered clusters were reported as unitary clusters without any separation between the items.

(Received for publication January 31, 1979.) 